\title{
A Case of Aspergillus Empyema Successfully Treated with Combination Therapy of Voriconazole and Micafungin: Excellent Penetration of Voriconazole and Micafungin into Pleural Fluid
}

\author{
Toshiaki Matsuda, Yoshimizu Koreeda, Hiroko Mataki, Tetsuhiko Taira, \\ Satoshi Noma and Ikkou Higashimoto
}

\begin{abstract}
A 62-year-old man with empyema caused by Aspergillus fumigatus was successfully treated with a combination of voriconazole (VRCZ) and micafungin (MCFG). Data regarding the penetration of antifungal agents into pleural fluid are limited. Thus, we measured the concentration of VRCZ and MCFG in his plasma and pleural fluid. Penetration of VRCZ and MCFG into the pleural fluid was excellent. Therefore, the combination therapy using VRCZ and MCFG may contribute to successful management of Aspergillus empyema.
\end{abstract}

Key words: Aspergillus fumigatus, empyema, voriconazole, micafungin, pleural fluid

(Inter Med 49: 1163-1169, 2010)

(DOI: 10.2169/internalmedicine.49.2860)

\section{Introduction}

Aspergillus empyema is a rare and potentially lifethreatening invasive fungal infection(1). Aspergillus empyema is a complication of aspergilloma and chronic necrotizing pulmonary aspergillosis (CNPA) or surgery for these diseases and responds to treatment slowly $(2,3)$. Some cases reported in the literature were treated with standard regimens for invasive pulmonary aspergillosis (IPA) or underwent surgical therapy (4-7). Three classes of antifungal drugs are available for treating aspergillosis: polyenes, azoles, and echinocandins $(8,9)$. Recently, voriconazole (VRCZ), an azole antimycotic agent, was recommended as a primary therapeutic agent for invasive aspergillosis (9). For refractory cases, addition of another drug for salvage therapy has been recommended in the guidelines for treating aspergillosis. Preclinical and clinical data have suggested a synergistic interaction between echinocandins and triazoles against Aspergillus species (10-12). In one study, micafungin (MCFG), used in primary or salvage therapy, was proven to be efficacious and safe for high-risk patients with IPA, although the number of patients studied was small (13).
Adequate drug penetration into the infection site is crucial for optimal antimicrobial treatment. Although there are many studies regarding penetration of antibacterial agents into pleural effusion (14), there are only a few reports describing penetration of antifungal agents into pleural fluid in empyema cases $(15,16)$. To our knowledge, there are no reports regarding MCFG penetration into pleural space in empyema cases.

Here, we report a case of Aspergillus empyema caused by A. fumigatus. The patient was successfully treated using a combination of VRCZ and MCFG, following the guidelines for treating aspergillosis. To evaluate drug penetration into pleural fluid, we measured VRCZ and MCFG concentrations in plasma and pleural fluid using high performance liquid chromatography (HPLC). Here, we defined the penetration ratio as follows: antifungal agent concentration in pleural fluid sample/simultaneous antifungal agent level in the plasma sample, expressed as a percentage.

\section{Case Report}

In May 2007, a 62-year-old Japanese man had complained of increased shortness of breath upon exertion. His 


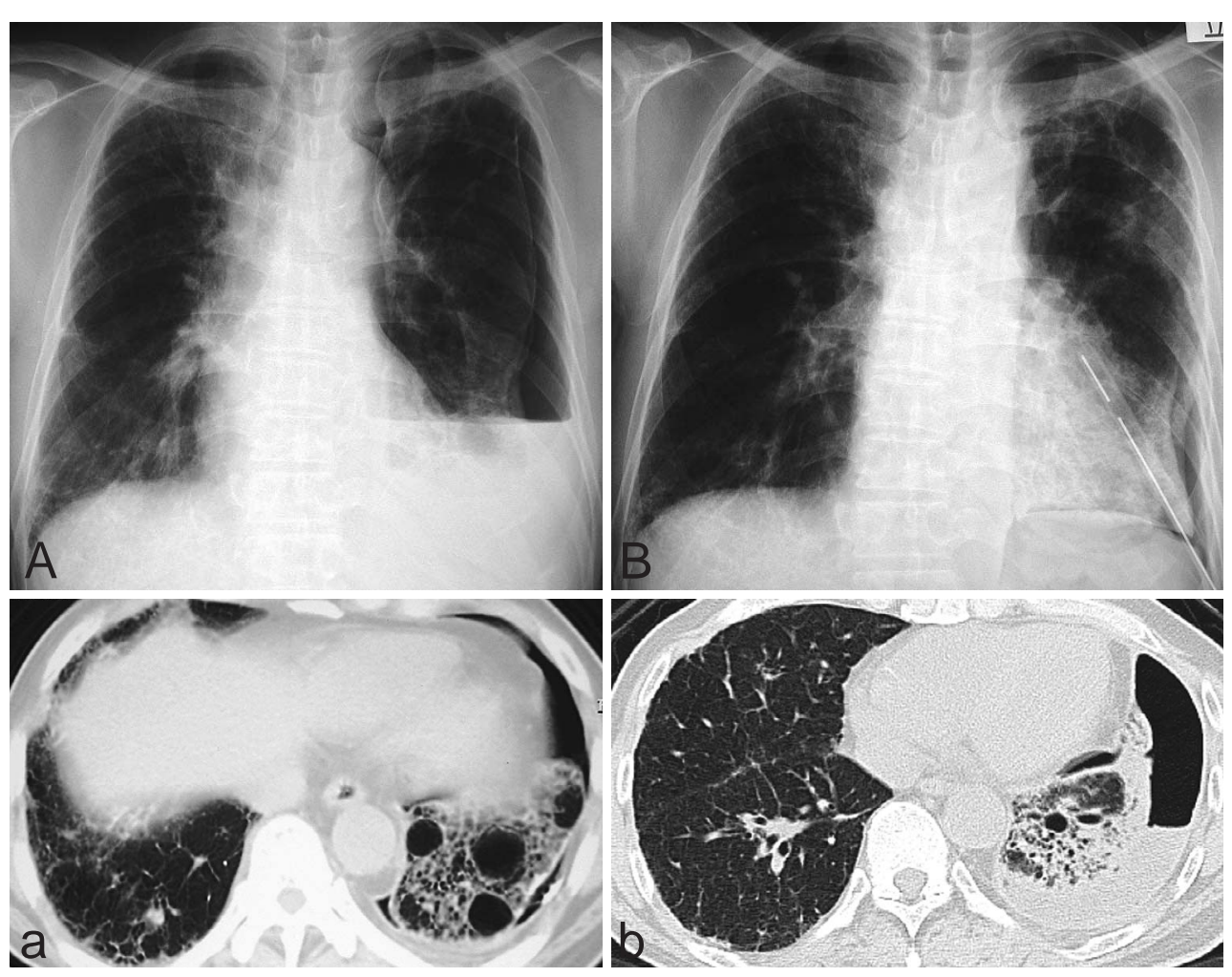

Figure 1. Chest X-ray (CXR) at the regular hospital (A) shows pneumothorax with air-fluid level in the left thoracic cavity; chest CT (a) shows collapse of the left lung with bulla. CXR on the day of admission to our hospital (B) shows inserted drainage tube and pneumothorax in the left thoracic space; chest CT shows collapsed lung with visceral pleural thickening and pleural fluid in the left thoracic cavity (b).

medical history was notable for rheumatoid arthritis (RA), interstitial pneumonia (IP), diabetes mellitus, and idiopathic thrombocytopenic purpura. He was diagnosed with acute exacerbation of IP in 2005. At that time, steroid pulse therapy, followed by maintenance therapy [FK506 for 4 months and prednisolone (PSL)], was administered to him. He was prescribed a daily maintenance dose of $5 \mathrm{mg}$ PSL, since March 2006. At his regular hospital, a chest X-ray and computed tomography (CT) scan revealed an air-fluid level in the left pleural space (Figs. 1A, 1a). He was admitted to the hospital, given chest tube drainage, and received a preliminary diagnosis of empyema and pneumothorax. Laboratory findings for pleural fluid in the first hospital were as follows: flank pus; white blood cell (WBC) count, 3,000/ $\mu \mathrm{L}$ with $80 \%$ neutrophils; lactate dehydrogenase (LDH), 4,308 IU/L; total protein (TP), $8.3 \mathrm{~g} / \mathrm{dL}$; and adenosine deaminase (ADA), 93 IU/L. Pleural fluid culture tests for bacteria and mycobacteria were negative. Despite chest tube drainage and systemic administration of antibiotics for 6 weeks, purulent pleural fluid and high fever persisted. He was then transferred to our hospital with a diagnosis of refractory empyema.

On the day of admission, his vital signs were as follows: blood pressure, $126 / 70 \mathrm{mmHg}$; heart rate, 75 beat per min; respiratory rate, 15 breaths per min; and body temperature, $36.7^{\circ} \mathrm{C}$. Physical examination revealed slightly anemic conjunctiva, fine crackles on the right side of the lung field, and pleural friction rub on the left side of the lung. There was no deformity, pain, tenderness, swelling, or redness in any joints. Laboratory findings on the day of admission were as follows: WBC, $6,920 / \mu \mathrm{L}$ with $86 \%$ neutrophils; $11 \%$ lymphocytes, $2 \%$ monocytes, $75 \% \mathrm{~T}$ cells, $25 \% \mathrm{CD} 4 \mathrm{~T}$ cells, 49\% CD8 T cells, and 16\% natural killer cells; C-reactive protein (CRP), $12.7 \mathrm{mg} / \mathrm{dL}$; erythrocyte sedimentation rate, $140 \mathrm{~mm} / \mathrm{h}$; LDH, $157 \mathrm{IU} / \mathrm{L}$; TP, $7.1 \mathrm{~g} / \mathrm{dL}$; rheumatoid factor (RF), $17.5 \mathrm{IU} / \mathrm{mL}$; IgG, 2,733 mg/dL; IgM, $110 \mathrm{mg} / \mathrm{dL}$; IgA, $311 \mathrm{mg} / \mathrm{dL}$; anti-cyclic citrullinated peptide antibody, $36 \mathrm{U} / \mathrm{mL}$; anti-galactose defect IgG antibody, $64 \mathrm{AU} / \mathrm{mL}$; matrix metalloproteinase-3, $104.1 \mathrm{ng} / \mathrm{mL}$; platelet-associated IgG, 285 ng/10 cells; KL-6, $82 \mathrm{U} / \mathrm{mL}$; SP-D, $117 \mathrm{ng} / \mathrm{mL}$; and $(1,3)-\beta$-D-glucan, $14.8 \mathrm{pg} / \mathrm{mL}$. The QuantiFERON TB$2 \mathrm{G}$, human immunodeficiency virus test, galactomannan antigen test, and anti-Aspergillus antibody test were negative. A second chest X-ray and CT scan (Figs. 1B, 1b) revealed pleural fluid, lung volume reduction, thickened pleura, and left side pneumothorax. A sputum culture test revealed normal flora and was negative for mycobacteria and fungi, including Aspergillus species. Pleural fluid examination revealed yellowish purulent features: WBC, 180,860/ $\mu \mathrm{L}$ with 98\% neutrophils; LDH, 11,690 IU/L; pH, 7.215; TP, 4.9 g/ $\mathrm{dL}$; glucose, $0 \mathrm{mg} / \mathrm{dL}$; RF, <2.0 IU/mL; carcinoembryonic antigen, $0.9 \mathrm{ng} / \mathrm{mL}$; and $\mathrm{ADA}, 94 \mathrm{IU} / \mathrm{L} / 37^{\circ} \mathrm{C}$. Cytological examination for malignancy was negative. Pleural fluid culture tests for bacteria and mycobacteria were negative. Polymerase chain reaction (PCR) test of pleural fluid for $M y$ - 

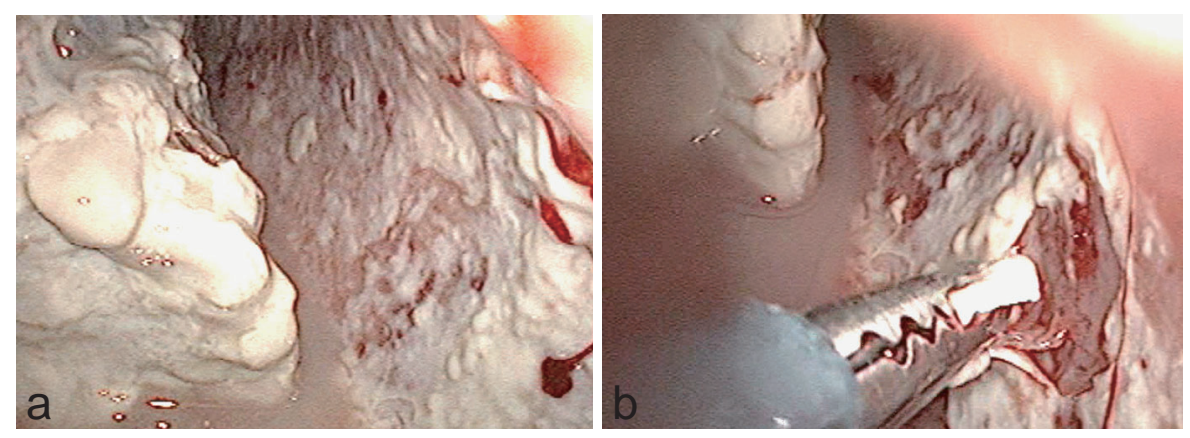

Figure 2. Intra-thoracic finding of video-assisted thoracoscopy for diagnostic purpose (a, b); visceral and parietal pleura covered with white, thick, soft caseous necrotic tissues.
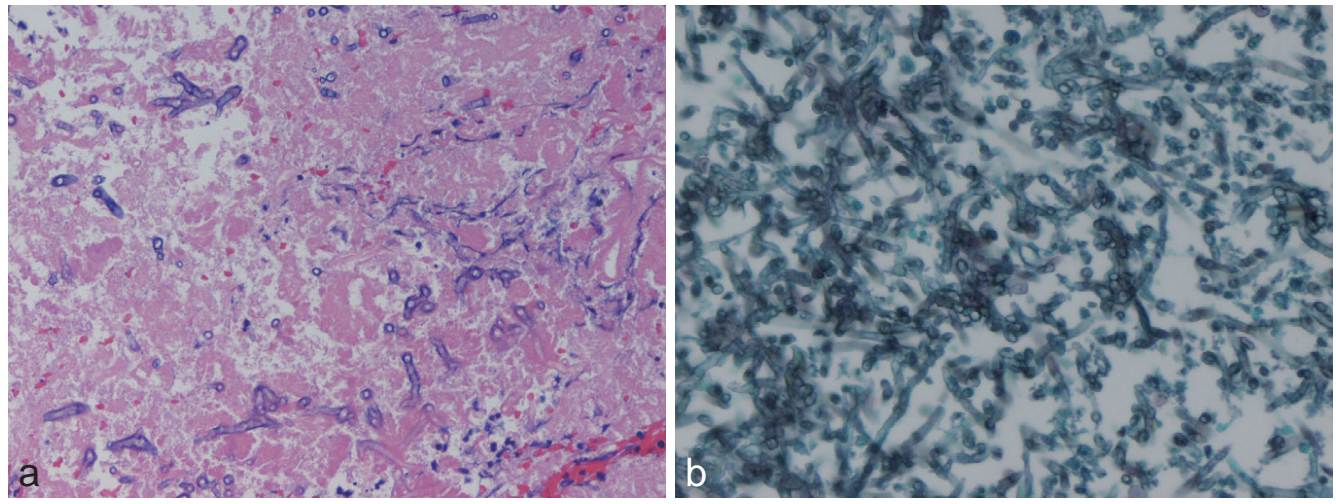

Figure 3. Pathological finding shows narrow, acutely branching septate hyphae in necrotic tissue on Hematoxylin Eosin staining and Grocott's stain (a, b).

cobacterium tuberculosis was negative. Because pleural fluid culture resulted in the growth of Aspergillus species, Aspergillus empyema was suspected.

The chest tube that had been placed in the left side of the pleural space for 7 weeks was removed, and antifungal therapy comprising oral VRCZ [300 mg twice daily (day 1)] followed by a maintenance dose of $150 \mathrm{mg}$ twice daily, was administered. However, spiking fever and increasing pleural fluid persisted. For diagnostic purposes, we performed exploratory video-assisted thoracic surgery, which revealed parietal and visceral pleura enveloped by white necrotizing tissues (Figs. 2a, 2b).

Histopathological examination of a pleural biopsy (Figs. 3a, 3b) revealed branching septate hyphae, and their culture resulted in the growth of A. fumigatus, providing for a definitive diagnosis of Aspergillus empyema. Isolation of A. fumigatus and susceptibility tests were performed at First Laboratories, Co., Ltd., Medical Mycology Research Center, Chiba University, Chiba, Japan. A. fumigatus identification was established by determining the internal transcribed spacer region of the rRNA gene. Minimum inhibitory concentrations (MICs) of antifungal agents against A. fumigatus were as follows: amphotericin (AMPH), $0.25 \mu \mathrm{g} / \mathrm{mL}$; flucytosine, $1 \mu \mathrm{g} / \mathrm{mL}$; fluconazole, $>64 \mu \mathrm{g} / \mathrm{mL}$; itraconazole, 0.12 $\mu \mathrm{g} / \mathrm{mL}$; miconazole, $0.5 \mu \mathrm{g} / \mathrm{mL}$; MCFG, $\leq 0.03 \mu \mathrm{g} / \mathrm{mL}$; and VRCZ, $0.5 \mu \mathrm{g} / \mathrm{mL}$. Bronchoscopic examination revealed no abnormalities in the trachea or bronchus. Bronchial lavage fluid culture revealed normal flora and was negative for Aspergillus species. The pleural fluid tests and clinical course excluded other possible conditions, such as malignancy, tuberculosis, and rheumatoid pleuritis. All pleural fluid culture tests for bacteria were negative, and the diagnosis of bacterial empyema was not established. Purulent pleural fluid and increased levels of serum CRP and $(1,3)-\beta$-D-glucan persisted, although VRCZ concentrations in the serum and pleural fluid were higher than their MICs for A. fumigatus. In response to this, we increased the dose of VRCZ to 300 $\mathrm{mg}$ twice daily. However, purulent pleural effusion and laboratory data including CRP and (1,3)- $\beta$-D-glucan did not improve, so we assumed that the patient had refractory empyema. Intravenous MCFG $150 \mathrm{mg}$ daily for 90 days (day 25 to 115 of VRCZ administration) was added to the antifungal regimen as salvage therapy. Clinical symptoms and laboratory findings gradually improved and the follow-up pleural fluid culture was negative (Fig. 4). When the pleural fluid drainage volume decreased to $<50 \mathrm{~mL} /$ day, the chest tube was removed; this was on day 43 after VRCZ administration (Fig. 5). Abnormal liver function tests (LFTs) and an abrupt increase in plasma VRCZ trough level occurred on day 95 after VRCZ administration, and we adjusted the VRCZ dose to $250 \mathrm{mg}$ twice daily. LFTs promptly improved. The patient was discharged after discontinuing MCFG administration. He was maintained with oral VRCZ over a 13-month period. No clinical signs of recurrence, 
2007
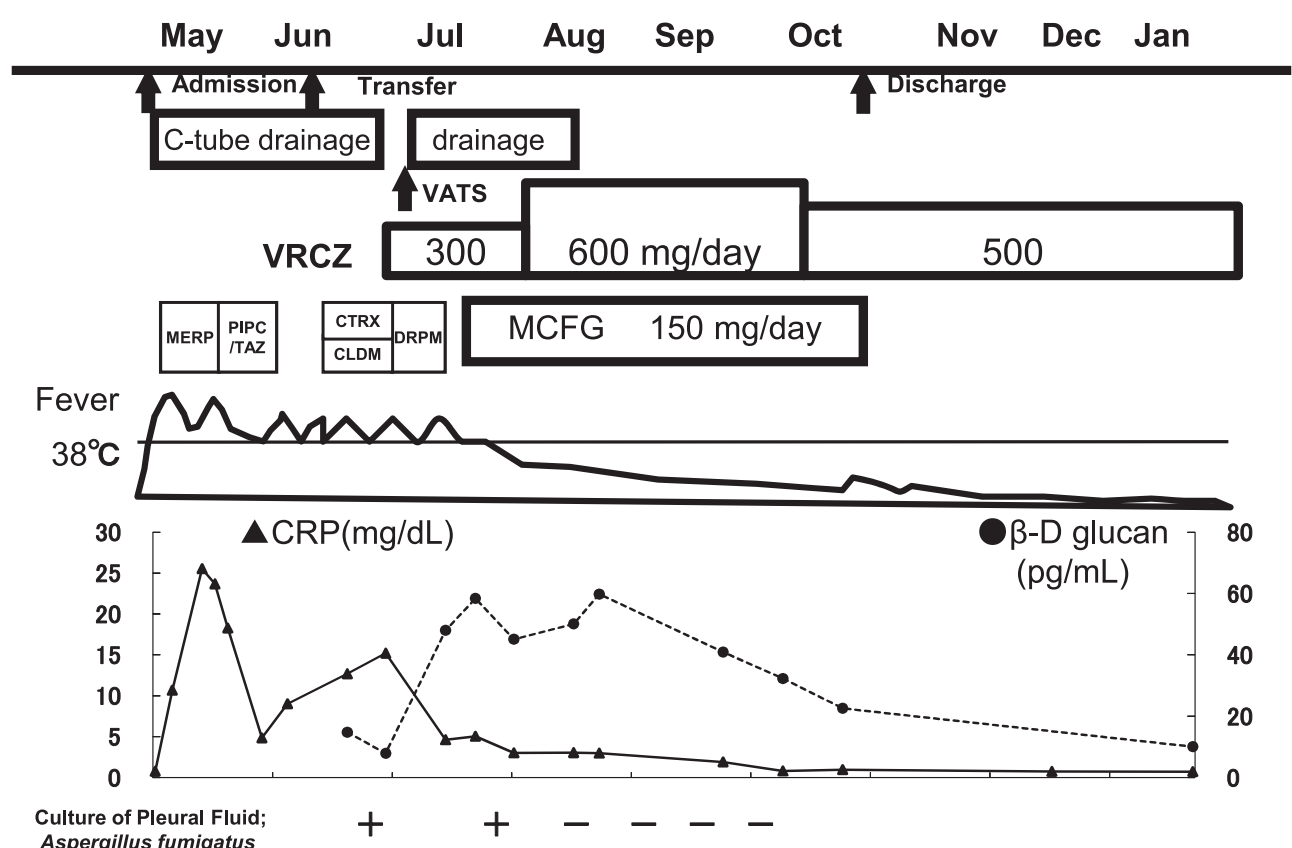

Figure 4. Clinical course.

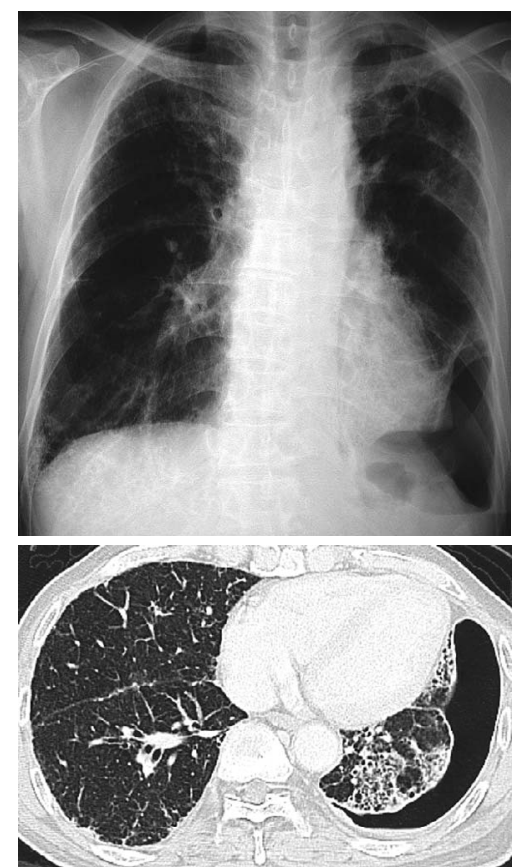

Figure 5. Chest X-ray and CT, after discontinuation of chest tube drainage, show collapsed lung and decreased pleural fluid in the left thoracic cavity.

such as increasing pleural fluid, (1,3)- $\beta$-D-glucan, CRP, galactomannan antigen, or anti-Aspergillus antibody, were noted after discontinuation of antifungal therapy during the follow-up period.

\section{Discussion}

Here, we described a case of Aspergillus empyema caused by A. fumigatus, successfully treated with a combination of
VRCZ and MCFG. We measured the concentration of VRCZ and MCFG in plasma and pleural fluid. Penetration of VRCZ and MCFG into pleural fluid was excellent (Tables 1,2$)$.

Adequate drug penetration into an infection site is crucial for optimal antimicrobial therapy. In general, the pleural surface is thicker and more acidic in patients with empyema. Pleural diffusion of antibiotics is highly variable (14). There are only a few reports regarding the penetration of antifungal agents, such as VRCZ, and AMPH B lipid formulations into pleural fluid $(15,16)$. In the report of VRCZ penetration into pleural fluid (15), trough concentrations of plasma and pleural fluid were $0.95-1.4 \mu \mathrm{g} / \mathrm{mL}$ and $0.9-1.2 \mu \mathrm{g} / \mathrm{mL}$, respectively, in response to $\mathrm{VRCZ}$ at $200-300 \mathrm{mg}$ twice daily. The penetration ratio of $\mathrm{VRCZ}$ was $45-95 \%$. The authors concluded that excellent VRCZ penetration into pleural fluid appeared to contribute to the successful management of Aspergillus empyema caused by A. fumigatus. In contrast, Weiler et al reported that the penetration ratio into the pleural effusion of AMPH B lipid formulations was 3$44 \%$ in critically ill patients (16) and that long-term treatment with a high dose of AMPH B lipid formulations was required to eradicate fungal infections from pleural effusions. They concluded that alternative therapeutic strategies need to be considered. Thus far, there are limited data regarding penetration of antifungal agents into pleural effusion.

We monitored VRCZ concentrations in plasma and pleural fluid $2 \mathrm{~h}$ after oral intake $(\mathrm{C} 2)$ and at trough concentrations $(\mathrm{C} 0)$ using HPLC. In the present case, trough concentrations of plasma and pleural fluid were $3.2-6.5 \mu \mathrm{g} / \mathrm{mL}$ and $0.7-4.4 \mu \mathrm{g} / \mathrm{mL}$, respectively, in response to VRCZ at 200$300 \mathrm{mg}$ twice daily (Table 1). Pleural culture test revealed 
Table 1. VRCZ Concentrations in Plasma and Pleural Fluid

\begin{tabular}{|c|c|c|c|c|c|c|}
\hline \multirow{3}{*}{ Day of VRCZ (dose) } & \multicolumn{4}{|c|}{ VRCZ concentration $(\mu \mathrm{g} / \mathrm{mL})$ in: } & \multirow{2}{*}{\multicolumn{2}{|c|}{$\begin{array}{c}\text { Penetration ratio } \\
(\%)\end{array}$}} \\
\hline & \multicolumn{2}{|c|}{ Plasma } & \multicolumn{2}{|c|}{ Pleural fluid } & & \\
\hline & C 0 & C 2 & $\mathrm{CO}$ & C2 & Co & C2 \\
\hline 5 (150 mg twice a day) & 2.9 & & & & & \\
\hline 16 (200 mg twice a day) & 3.2 & 3.7 & 0.7 & 1.4 & 19 & 38 \\
\hline 24 (300 mg twice a day) & 6.1 & 7.1 & 1.4 & 2.1 & 23 & 29 \\
\hline 33 (300 mg twice a day) & 6.5 & 7.4 & & & & \\
\hline 47 (300 mg twice a day) & 6.5 & 7.1 & & & & \\
\hline 54 (300 mg twice a day) & $5.8^{*}$ & $6.7^{*}$ & $4.4^{*}$ & & $75^{*}$ & \\
\hline 68 (300 mg twice a day) & $6.1^{*}$ & $7.2^{\star}$ & $4.3^{*}$ & & $70^{*}$ & \\
\hline 82 (300 mg twice a day) & $5.7^{\star}$ & $6.6^{\star}$ & & & & \\
\hline 96 (300 mg twice a day) & $9.1^{*}$ & $12.0^{*}$ & & & & \\
\hline 110 (250 mg twice a day) & $6.0^{\star}$ & $6.3^{*}$ & & & & \\
\hline
\end{tabular}

$\mathrm{CO}$ : concentration at trough; $\mathrm{C} 2$ : concentration at $2 \mathrm{~h}$ after oral intake. * data in the setting without chest tube drainage.

Table 2. MCFG Concentrations in Plasma and Pleural Fluid

\begin{tabular}{cccc}
\hline \multirow{2}{*}{ Day of MCFG } & \multicolumn{2}{c}{ MCFG concentration $(\mu \mathrm{g} / \mathrm{mL})$ in: } & $\begin{array}{c}\text { Penetration } \\
\text { ratio (\%) }\end{array}$ \\
\cline { 2 - 3 } & Plasma $(\mathrm{C22})$ & Pleural fluid (C22) & $57^{\star}$ \\
\hline Day 29 & $4.42^{*}$ & $2.52^{\star}$ & $67^{\star}$ \\
\hline Day 43 & $5.36^{\star}$ & $3.61^{\star}$ & \\
\hline
\end{tabular}

C22: Concentration at $22 \mathrm{~h}$ after the last dose.

${ }^{*}$ data in the setting without chest tube drainage.

that the VRCZ concentration in pleural fluid was higher than its MIC for A. fumigatus. The penetration ratio of VRCZ was $19-75 \%$, which was lower than that previously reported by Stern et al (15). Our results suggest that chest tube drainage decreases the pleural fluid VRCZ concentration. VRCZ accumulation in the pleural fluid occurred after chest tube drainage was discontinued. In the setting without chest tube drainage, the penetration ratio of VRCZ into pleural fluid was 70-75\%.

To our knowledge, there are no reports regarding MCFG penetration into the pleural space in empyema cases. To evaluate MCFG penetration into pleural fluid, MCFG levels in plasma and pleural fluid were also measured by HPLC. In response to MCFG (150 mg daily), concentrations of MCFG in plasma and pleural fluid at $22 \mathrm{~h}$ after the last dose were 4.37-5.25 $\mu \mathrm{g} / \mathrm{mL}$ and 2.50-3.58 $\mu \mathrm{g} / \mathrm{mL}$, respectively (Table 2). The penetration ratio of MCFG was 57$68 \%$. Data of the MCFG concentrations in plasma and pleural fluid were available only after discontinuing chest tube drainage. Pleural culture test revealed that the MCFG con- centration in pleural fluid was higher than its MIC for $A$. $f u$ migatus. It may contribute to successful management of $A s$ pergillus empyema.

According to the guideline for aspergillosis treatment, primary combination therapy for IPA is not routinely recommended, because of the lack of clinical data; instead, salvage therapy is recommended for refractory cases (9). Preclinical and clinical data have suggested a synergistic interaction between echinocandins and triazoles against Aspergillus species (10-12). In addition to the synergistic effect in vitro, adequate penetration into pleural fluid of MCFG and VRCZ may be another advantage in the treatment of Aspergillus empyema. Although further studies of efficacy and safety are required, the combination of VRCZ and MCFG is suitable for treating Aspergillus empyema for these reasons.

Aspergillus empyema usually occurs as a complication of aspergilloma and CNPA or surgery for lung cancer, tuberculosis, or aspergillosis; it responds to treatment slowly $(2,7,17)$. In the present case, the source of A. fumigatus in the pleural space was of interest because there was 
no history of aspergillosis or surgical resection. Further, there were no obvious signs indicating pleura-pulmonary fistula, chronic cavity with fungus balls, or a new cavitation lesion on CT scan, although collapsed lung and pleural effusion made it difficult to evaluate abnormalities in the lung. However, we believe the patient had a primary pulmonary aspergillosis lesion which penetrated into the pleural space, and that this resulted in the occurrence of pneumothorax and empyema. First, the presence of infiltration and small cystic lesions may be consistent with the pneumonic process. Second, no organism, except for A. fumigatus, was detected in pleural effusion and plasma in this case. Culture tests of sputum and bronchial lavage were negative for bacteria, mycobacteria, and fungi. Third, the improvement of empyema with an antifungal regimen and drainage ruled out other conditions, such as malignancy and tuberculous pleuritis. An RF test of pleural fluid excluded the occurrence of rheumatoid pleuritis. Finally, our patient had risk factors for pulmonary aspergillosis, including steroid therapy.

The usual route of infection for invasive aspergillosis is through inhalation of Aspergillus conidia into the lungs. Patients with altered immunity, particularly those with reduced pulmonary defense, which inhibits activities of pulmonary macrophages (such as those using corticosteroids), or those who are neutropenic, have increased susceptibilities to Aspergillus species (18-20). Takayanagi et al reported 12 patients with pulmonary aspergillosis in a study of 149 RA patients with pulmonary infections (21). They concluded that pre-existing lung lesions and low-dose corticosteroids (PSL $\leq 10 \mathrm{mg}$ ) were risk factors for pulmonary aspergillosis. The present patient was susceptible to Aspergillus infection because of risk factors that included a pre-existing lung lesion, corticosteroid therapy, and type 2 diabetes mellitus, although he was not neutropenic.

Corticosteroids play a major role in increasing susceptibility to Aspergillus by inhibiting oxidative killing of microorganisms by pulmonary macrophages (22). Hydrocortisone significantly increases growth rates of Aspergillus species (23). Although the use of corticosteroids is a risk factor for Aspergillus infections, we retained the PSL dose during treatment of empyema in order to avoid any influence of RA and IP disease activities. Fortunately, these baseline diseases were stable. However, even a low PSL dose may cause empyema to become refractory, as was observed in this case.

In this case, depletion of total lymphocyte counts and CD $4 \mathrm{~T}$ cell counts was present on admission. Lymphocytopenia associated with most acute infectious disease is usually mild and rapidly reversed, and in the present case, the lymphocytopenia and lymphocyte population abnormality recovered with antifungal treatment of empyema.

In conclusion, we successfully treated a patient with refractory empyema caused by A. fumigatus using combination therapy consisting of VRCZ and MCFG. In the present case, VRCZ and MCFG achieved pleural fluid concentrations greater than their MICs for A. fumigatus; thus, excellent penetrations of VRCZ and MCFG into pleural fluid were demonstrated. This may account for the successful management of this infection.

\section{Acknowledgement}

We would like to thank Dr. Kazuko Nishimura, Professor Emeritus, Medical Mycology Research Center, Chiba University, for isolating and identifying Aspergillus fumigatus and performing the susceptibility tests. We are also indebted to Kazuaki Matsumoto, PhD; Kazuko Abematsu; Yasuo Takeda, PhD; and Katsushi Yamada, PhD, Professor, Department of Clinical Pharmacy and Pharmacology, Kagoshima University Hospital, for monitoring plasma and pleural fluid concentrations of VRCZ; and to Saori Nakagawa, PhD; Susumu Yamato, PhD, Professor, Department of Bio-Analytical Chemistry, Faculty of Pharmaceutical Sciences, Niigata University of Pharmacy and Applied Life Sciences, for measuring plasma and pleural fluid MCFG concentrations. We are grateful to Dr. Kazuhiro Tabata, Department of Human Pathology, Kagoshima University Graduate School of Medical and Dental Sciences, for pathologic diagnosis. And lastly, we would like to thank Dr. Yoshihiro Nakamura, Department of Thoracic and Cardiovascular Surgery, Kagoshima University Hospital, for his help in the exploratory video-assisted thoracic surgery for diagnostic purposes.

\section{References}

1. Ko SC, Chen KY, Hsueh PR, Luh KT, Yang PC. Fungal empyema thoracis: an emerging clinical entity. Chest 117: 1672-1678, 2000.

2. Denning DW. Chronic forms of pulmonary aspergillosis. Clin Microbiol Infect 7 (Suppl 2): 25-31, 2001.

3. Shiraishi Y, Katsuragi N, Nakajima Y, Hashizume M, Takahashi N, Miyasaka Y. Pneumonectomy for complex aspergilloma: is it still dangerous? Eur J Cardiothorac Surg 29: 9-13, 2006.

4. Shirakusa T, Ueda H, Saito T, Matsuba K, Kouno J, Hirota N. Surgical treatment of pulmonary aspergilloma and Aspergillus empyema. Ann Thorac Surg 48: 779-782, 1989.

5. Lodge BA, Ashley ED, Steele MP, Perfect JR. Aspergillus fumigatus empyema, arthritis, and calcaneal osteomyelitis in a lung transplant patient successfully treated with posaconazole. J Clin Microbiol 42: 1376-1378, 2004.

6. Mukae H, Iwamoto M, Tagawa H, et al. A case of Aspergillus em- pyema with bronchopleural fistula. Nihon Kyobu Shikkan Gakkai Zasshi 28: 1482-1487, 1990 (in Japanese, Abstract in English).

7. Lampo N, Spiliopoulos A, Licker M, Tschopp JM. Management of postpneumonectomy Aspergillus empyema extending into the thoracic wall: a plea for radical surgery and caution when using liposomal amphotericin B. Interact Cardiovasc Thorac Surg 2: 682-684, 2003.

8. Segal BH. Aspergillosis. N Engl J Med 360: 1870-1884, 2009.

9. Walsh TJ, Anaissie EJ, Denning DW, et al. Treatment of aspergillosis: clinical practice guidelines of the Infectious Diseases Society of America. Clin Infect Dis 46: 327-360, 2008.

10. Marr KA, Boeckh M, Carter RA, Kim HW, Corey L. Combination antifungal therapy for invasive aspergillosis. Clin Infect Dis 39: 797-802, 2004

11. Heyn K, Tredup A, Salvenmoser S, Mulller FM. Effect of vori- 
conazole combined with micafungin against Candida, Aspergillus, and Scedosporium spp. and Fusarium solani. Antimicrob Agents Chemother 49: 5157-5159, 2005.

12. Lewis RE, Kontoyiannis DP. Micafungin in combination with voriconazole in Aspergillus species: a pharmacodynamic approach for detection of combined antifungal activity in vitro. J Antimicrob Chemother 56: 887-892, 2005.

13. Denning DW, Marr KA, Lau WM, et al. Micafungin (FK463), alone or in combination with other systemic antifungal agents, for the treatment of acute invasive aspergillosis. J Infect 53: 337-349, 2006.

14. Teixeira LR, Sasse SA, Villarino MA, Nguyan T, Muligan ME, Light RW. Antibiotic levels in empyemic pleural fluid. Chest 117: 1734-1739, 2000.

15. Stern JB, Girard P, Caliandro R. Pleural diffusion of voriconazole in a patient with Aspergillus fumigatus empyema thoracis. Antimicrob Agents Chemother 48: 1065, 2004.

16. Weiler S, Bellmann-Weiler R, Joannidis M, Bellmann R. Penetration of amphotericin B lipid formulations into pleural effusion. Antimicrob Agents Chemother 51: 4211-4213, 2007.

17. Wex P, Utta E, Drozdz W. Surgical treatment of pulmonary and pleuro-pulmonary Aspergillus disease. Thorac Cardiovasc Surg 41:
64-70, 1993.

18. Walsh TJ, Dixon DM. Nosocomial aspergillosis: environmental microbiology, hospital epidemiology, diagnosis and treatment. Eur J Epidemiol 5: 131-142, 1989.

19. Walsh TJ, Pappas P, Winston DJ, et al. Voriconazole compared with liposomal amphotericin B for empirical antifungal therapy in patients with neutropenia and persistent fever. N Engl J Med 346: 225-234, 2002.

20. Thomas FP. Aspergillus species. In: Principles and Practice of Infectious Disease. 6th ed. Mandell GL, Bennett JE, Dolin R, Eds. Churchill Livingstone, 2005: 2958-2973.

21. Takayanagi N, Tsuchiya $Y$, Tokunaga D, et al. Pulmonary infections in patients with rheumatoid arthritis. Nihon Kokyuki Gakkai Zasshi 45: 465-473, 2007 (in Japanese, Abstract in English).

22. Schaffner A. Therapeutic concentrations of glucocorticoids suppress the antimicrobial activity of human macrophages without impairing their responsiveness to gamma interferon. J Clin Invest 76: $1755-1764,1985$.

23. Ng TT, Robson GD, Denning DW. Hydrocortisone-enhanced growth of Aspergillus spp.: implications for pathogenesis. Microbiology 140 (Pt 9): 2475-2479, 1994.

(C) 2010 The Japanese Society of Internal Medicine http://www.naika.or.jp/imindex.html 